\title{
High-resolution MRI of a peroneal intraneural ganglion cyst arising from the knee joint: illustrative case
}

\author{
Brandon W. Smith, MD, ${ }^{1}$ Megan M. Jack, MD, PhD, ${ }^{1}$ Garret M. Powell, MD, ${ }^{2}$ Matthew A. Frick, MD, ${ }^{2}$ Kimberly K. Amrami, MD, ${ }^{2}$ and \\ Robert J. Spinner, $M D^{1}$
}

Departments of ${ }^{1}$ Neurological Surgery and ${ }^{2}$ Radiology, Mayo Clinic, Rochester, Minnesota

BACKGROUND The advancement of high-resolution imaging and increased clinical experience have led to an increased understanding of the formation and treatment of intraneural ganglion cysts. Nearly all intraneural ganglion cysts in the common peroneal nerve have been reported to arise from a joint connection to the superior tibiofibular joint. The authors have identified four cases of intraneural ganglion cysts arising from the knee joint itself; however, none of these reported cases were well described, documented, or illustrated with high-resolution imaging.

OBSERVATIONS Here the authors present the case of an intraneural ganglion cyst arising from the knee joint and causing intermittent weakness and pain. The articular branch to the knee joint was clearly demonstrated on high-resolution magnetic resonance imaging and confirmed at surgical exploration. The patient was treated with articular branch ligation and has had complete resolution of his symptoms without recurrence of the cyst on follow-up imaging.

LESSONS This case adds to the mounting evidence that intraneural cyst pathology is dependent on a connection to a synovial joint as stated in the unifying theory of intraneural cyst development.

https://thejns.org/doi/abs/10.3171/CASE21130

KEYWORDS intraneural ganglion cyst; knee joint; intraneural cyst development; peroneal neuropathy

Intraneural ganglion cysts are mucinous lesions within the epineurium of nerves that occur in para-articular locations. Once considered curious entities, they are now well characterized. ${ }^{1-3}$ The use of high-resolution imaging in patients with these cysts has led to further understanding of the pathogenesis. A joint connection is a constant finding, and imaging patterns are described on the basis of this anatomical connection. ${ }^{2,4-6}$ These advances have helped direct surgical interventions to the joint connection and/or the joint of origin. $4,5,7,8$

Intraneural ganglion cysts occurring in unusual locations have fostered controversy about the articular origin, especially when joint connections are not immediately identified on imaging. ${ }^{4}$ We present a patient with intermittent foot drop who was found to have a peroneal intraneural ganglion cyst. Although the superior tibiofibular joint (STFJ) is the most common site for an intraneural cyst, this case differed in that the high-resolution imaging revealed the knee joint as the origin. We are unaware of a previous detailed description and illustration of this joint connection supported by preoperative magnetic resonance imaging (MRI) and operative findings.

\section{Illustrative Case}

A 49-year-old previously healthy man presented to us 5 weeks after noticing a "bump" over the lateral aspect of his left knee. Within a week of initially noticing the lesion, several additional subcutaneous masses were noted in this area. He complained of episodic numbness on the dorsum of the foot with intermittent foot drop. His symptoms were exacerbated by both running and deep knee bending, and he noted that the shape and size of the mass(es) changed with activities that involved knee flexion.

His examination revealed a prominent, superficially located, tubular-appearing, multilobulated cystic lesion within the common peroneal nerve in the popliteal fossa. The patient had full motor strength

ABBREVIATIONS MRI = magnetic resonance imaging; STFJ = superior tibiofibular joint. INCLUDE WHEN CITING Published May 24, 2021; DOI: 10.3171/CASE21130.

SUBMITTED February 25, 2021. ACCEPTED March 18, 2021.

(C) 2021 The authors, CC BY-NC-ND 4.0 (http://creativecommons.org/licenses/by-nc-nd/4.0/). 
in the lower limb, including the tibialis anterior, extensor digitorum, extensor hallucis longus, and peronei muscles. He was able to walk well on his heels. Percussion over the mass just above the popliteal crease produced radiating paresthesias on the dorsal foot. There was no Tinel's sign near the fibular tunnel. Evaluation included comparative MRI studies (1.5 T and $7 \mathrm{~T}$ ), which demonstrated a multilobulated peroneal intraneural cyst that ascended to the sciatic bifurcation with crossover to the tibial division of the sciatic nerve and a joint connection to the knee (Fig. 1).

With the patient prone and with tourniquet control, a curved incision was made in the popliteal fossa. The complex, multilobulated cyst was identified within the paraneurium of the common peroneal nerve and lateral sural cutaneous nerve. The intraneural cyst was evident to the point of the sciatic bifurcation, but not proximal to it (Fig. 2). A cystic nerve branch arose from the common peroneal nerve just above the popliteal crease. It looped around and passed deep to the popliteus muscle to enter the posterior knee joint (Fig. 2). This articular branch was ligated, and the cyst was decompressed in 3 points along the common peroneal
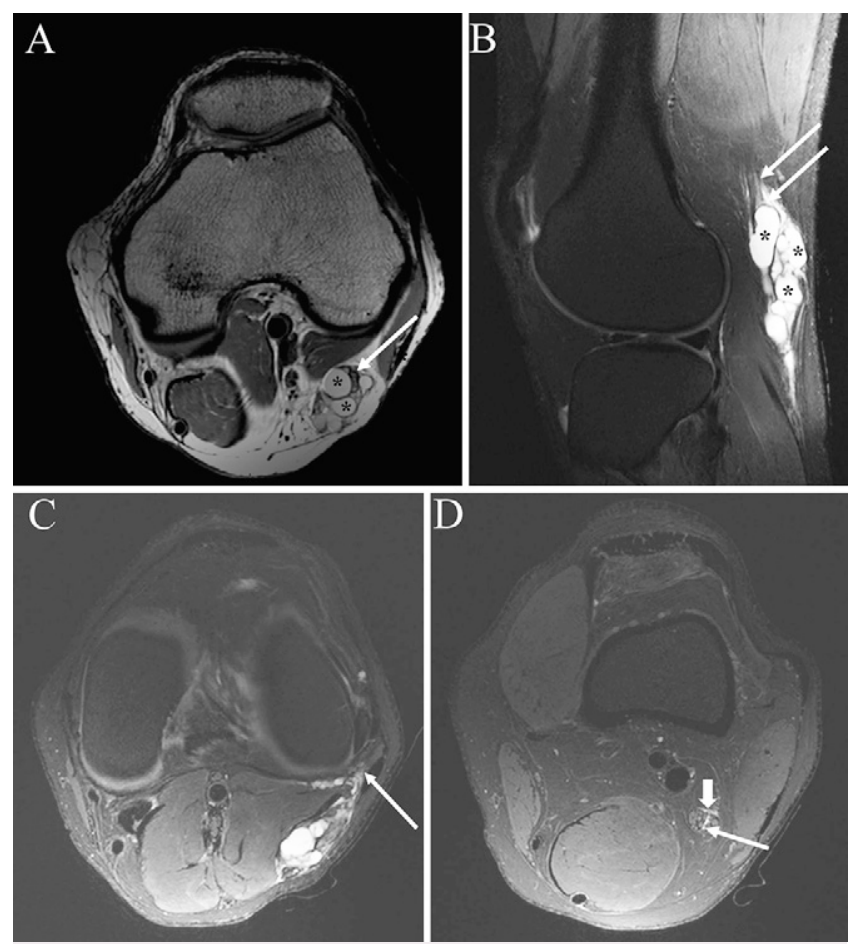

FIG. 1. Preoperative MRI. A: Axial proton density image of the knee demonstrating a complex intraneural (paraneurial) cyst (asterisks). The common peroneal nerve (arrow) shows increased T2 signal because of mass effect from the cyst. B: Sagittal T2-weighted MRI scan with fat suppression demonstrating the complexity of the intraneural cyst (asterisks) and the enlarged and hyperintense proximal common peroneal nerve. There is intraneural cyst extending proximally between nerve fascicles toward the bifurcation within the peroneal nerve (arrows). C: Axial T2-weighted MRI scan with fat suppression at the level of the knee joint shows the origin of the intraneural cyst from the posterolateral corner of the knee (arrow), communicating with the knee joint. D: Axial T2-weighted MRI study at the level of the sciatic bifurcation showing cyst within (long arrow) and around (short thick arrow) the nerve, confirming cyst crossover.
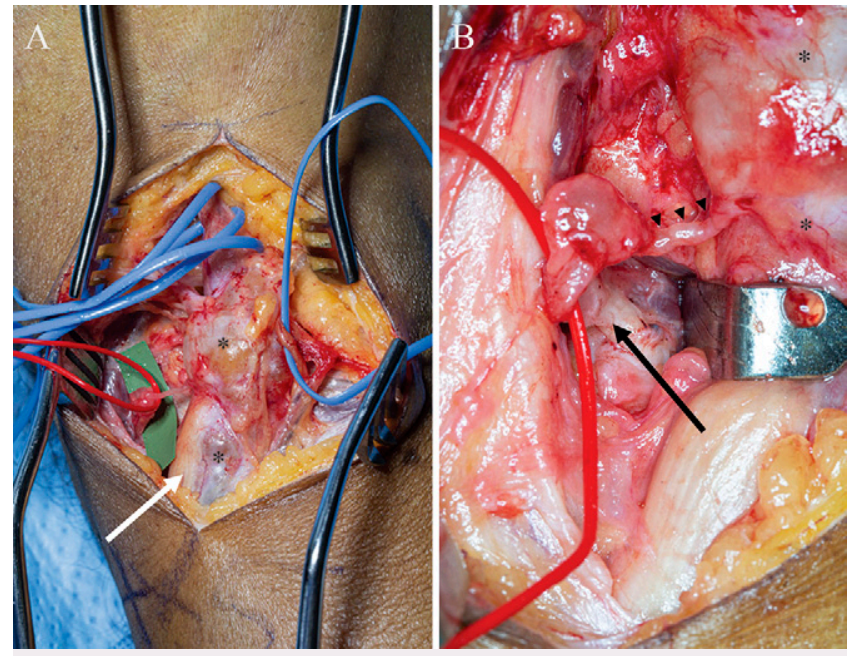

FIG. 2. The complex peroneal intraneural (paraneurial) cyst is seen (A, asterisks) around the common peroneal nerve. The common peroneal nerve is seen in blue vasoloops at the top of surgical exposure and below (white arrow). The sural communicating branch is seen in a blue vasoloop as well. The articular branch to the posterolateral knee joint is seen in the red vasoloop (A and B). The course of the serpiginous articular branch is seen (B). The black arrowheads show the small branch arising from the cyst (asterisk), then a cystic blowout and its clear joint connection (black arrow).

nerve to allow egress of the thick mucoid material. Given the patient's thin body habitus, a partial resection of the redundant cyst wall in the superficial layer was performed.

Postoperatively, the patient had full neurological function and resolution of his intermittent symptomatology. MRI completed 3 months postoperatively demonstrated decompression of the residual cyst wall with no evidence of recurrence (Fig. 3).

\section{Discussion}

\section{Observations}

Foot drop is a common condition with a broad differential diagnosis. ${ }^{9,10} \mathrm{MRI}$ and ultrasound are being used increasingly in the evaluation of patients with concern of having peripheral nerve lesions responsible for the deficit. In patients with common peroneal nerve lesions, the most frequent peripheral nerve localization of foot drop is the fibular neck region; intraneural ganglion cysts at this site are becoming more widely recognized on routine screening of patients with idiopathic peroneal neuropathy. ${ }^{11}$ Given its more common prevalence, the peroneal intraneural cyst at the fibular neck region has served as a prototype for research and discovery. The use of high-resolution imaging has helped to identify a joint connection in these patients to the anterior aspect of the STFJ. ${ }^{2,4,7,11}$ The articular theory was proposed and has been substantiated. Its fundamental principle is an articular branch-joint connection as the source of the intraneural ganglion at all sites of development. ${ }^{12,13}$

Peroneal intraneural ganglion cyst have, on rare occasions, been thought to originate from the knee joint. Only 4 of the 700 reported cases of peroneal intraneural ganglion cysts describe a knee origin ${ }^{14-17}$ as opposed to the typical site of origin, the STFJ. Three of these cases are included in larger series of peroneal cysts, ${ }^{15-17}$ 


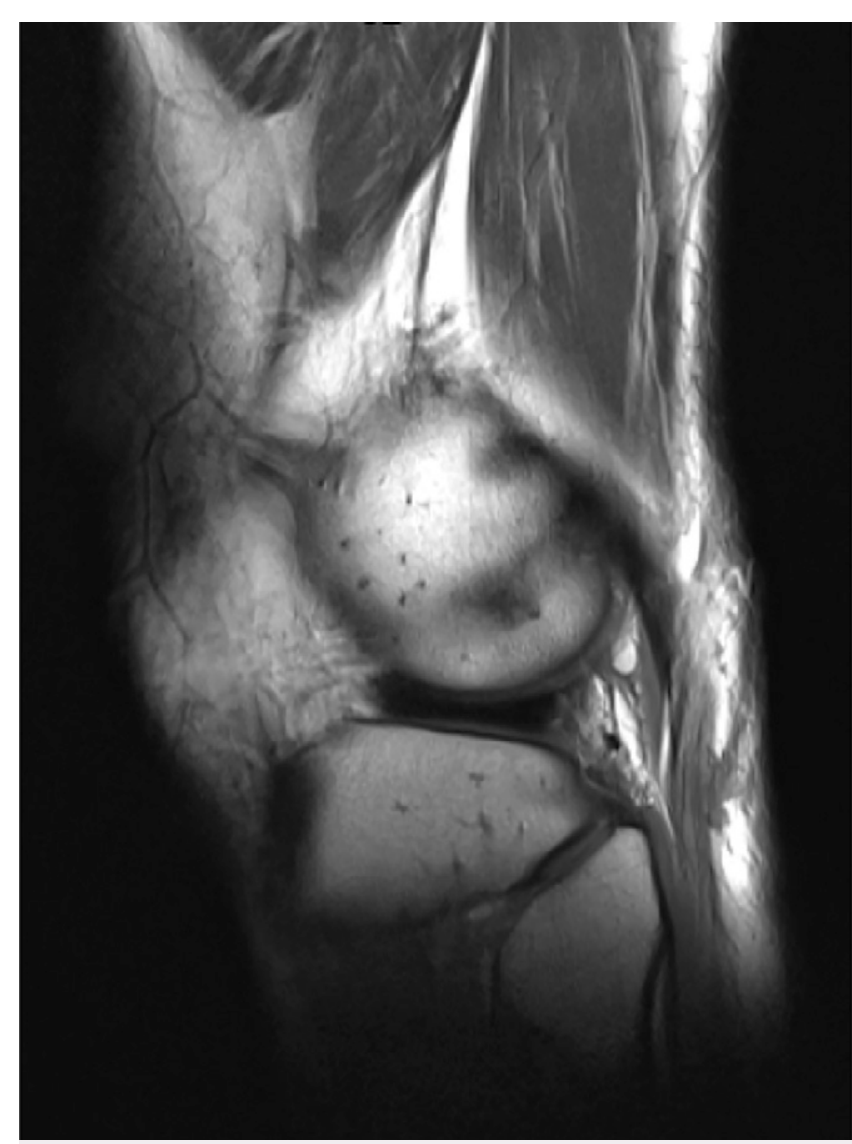

FIG. 3. MRI at 3 months after surgery demonstrating no residual or recurrence of the peroneal intraneural ganglion cyst arising from the articular branch from the knee joint.

and none of the reports is individually well described or illustrated, let alone characterized.

To understand the formation of intraneural ganglion cysts, we have relied on the foundational anatomical studies of the innervation of the joint capsule. The anatomy of the common peroneal nerve supports innervation to the STFJ with a clear articular branch that travels anterior to the fibular neck. This anatomy can be seen with pathognomonic findings on MRI and also intraoperatively. $4,5,18$ A common peroneal nerve connection to the posterior knee joint has also been well described. ${ }^{19}$ Branches to the posterior capsule of the knee can and, in this case, do serve as the conduit by which the joint fluid travels to the main nerve, creating an intraneural ganglion cyst. Thus, the occurrence of peroneal intraneural ganglion cysts in this location is perfectly understandable (Fig. 4). In this case as well as the other rare examples in the literature, a capsular defect in the knee joint allowed joint fluid to dissect in an articular branch, travel retrograde to the parent common peroneal nerve, and ascend to the sciatic nerve bifurcation. A peroneal intraneural ganglion cyst arising from the knee differs from that arising from the STFJ. It also has a characteristic appearance: The intraneural cyst occurs in the common peroneal nerve at the popliteal fossa, but not at the fibular neck, and originates through a small serpiginous connection to the posterior knee. Our case was a paraneurial form. ${ }^{20}$
Interestingly, we believe that one case of an extraneural ganglion cyst described in the supracondylar region is actually an intraneural (paraneurial) cyst arising from the knee joint. ${ }^{21}$

The occurrence of peroneal intraneural ganglion cysts arising from the knee joint (as well as from the STFJ) is directly analogous to the tibial intraneural ganglion cysts arising from the posterior knee joint and the posterior aspect of the STFJ. ${ }^{18,22}$ The articular branches that serve as the nerve connection between the joint and main nerve trunk are also described by Gardner. ${ }^{19}$ These small and often forgotten nerves to the posterior knee capsule can be the source of pathology affecting both the tibial and common peroneal nerves. This case provides further evidence to support that the articular theory applies to all cases of intraneural ganglion cysts, even when present in unusual locations. ${ }^{2}$

The articular basis for intraneural ganglion cysts has important treatment implications. In alignment with the treatment of peroneal intraneural cysts at the STFJ, we recommend that the intervention targets the cyst's joint of origin and not the cyst itself. Disconnection or ligation of the articular branch has been shown to have low morbidity and a favorably low recurrence rate. ${ }^{23,24}$ In contrast to the peroneal intraneural cyst arising from the STFJ, however, the knee joint does not lend itself to resection. ${ }^{18,24}$ Decompression of the cyst by itself without addressing the joint connection often leads to intraneural recurrence. On the basis of our experience with all types of cysts (intraneural and extraneural) arising from the STFJ, we do not perform an extensive resection of the cyst.

\section{Lessons}

There is mounting evidence that intraneural cysts have a common pathogenesis of a degenerative joint with an articular nerve branch acting as a conduit for fluid to propagate into the parent

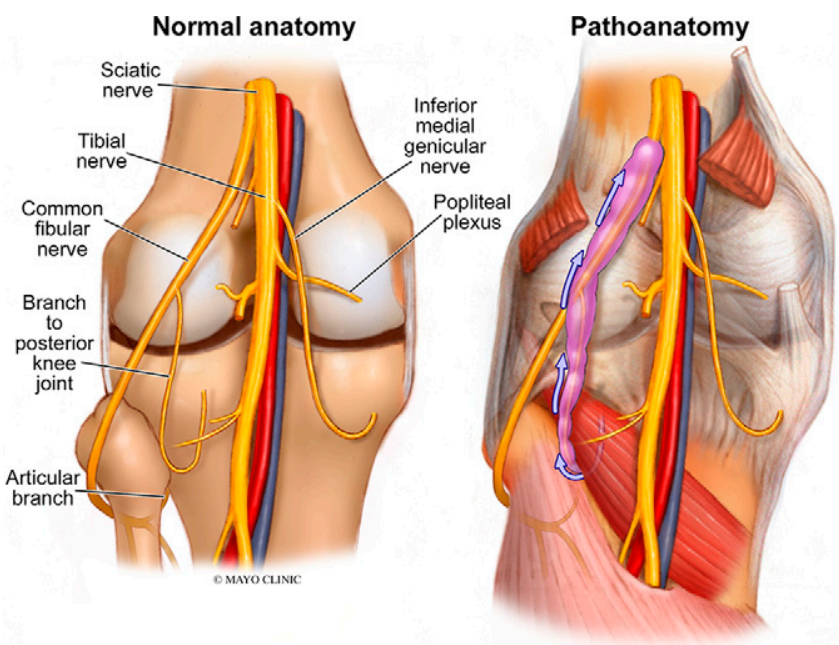

FIG. 4. Illustration of the normal anatomy of the posterior knee region and a comparison illustration showing the pathoanatomy noted in our patient. The peroneal intraneural cyst arising from the posterior knee and ascending (blue arrows) the articular branch and the common peroneal nerve to the sciatic bifurcation. The peroneal sural contribution noted in the surgical pictures was omitted for simplicity. Note the normal-appearing common peroneal nerve at the fibular tunnel (and articular branch to the STFJ). Reproduced with permission of the Mayo Clinic. 
nerve. The joint connection and/or the joint of origin should be the focus of therapy because cyst drainage in isolation is not adequate, resulting in unwanted intraneural recurrence, and extensive cyst resection is associated with unnecessary morbidity to the nerve. This case demonstrates a rare type of peroneal intraneural cyst in which the pathological articular branch arises from the posterior aspect of the knee joint rather than from the STFJ. In this variant, disconnection of the articular branch connection led to resolution of both the symptoms and the cyst itself; resection of the synovial surfaces, which has been performed for peroneal intraneural cysts arising from the STFJ, would not be feasible for this case arising from the knee joint.

\section{References}

1. Spinner RJ, Wang $H$, Angius $D$, et al. A modern perspective on the earliest description of an intraneural ganglion cyst arising from the knee joint. Clin Anat. 2011;24(1):106-112.

2. Spinner RJ, Scheithauer BW, Amrami KK. The unifying articular (synovial) origin of intraneural ganglia: evolution-revelation-revolution. Neurosurgery. 2009;65(suppl 4):A115-A124.

3. Desy NM, Wang H, Elshiekh MAl, et al. Intraneural ganglion cysts: a systematic review and reinterpretation of the world's literature. $J$ Neurosurg. 2016;125(3):615-630.

4. Spinner RJ, Luthra G, Desy NM, et al. The clock face guide to peroneal intraneural ganglia: critical "times" and sites for accurate diagnosis. Skeletal Radiol. 2008;37(12):1091-1099.

5. Spinner RJ, Desy NM, Amrami KK. The cystic transverse limb of the articular branch: a pathognomonic sign for peroneal intraneural ganglia at the superior tibiofibular joint. Neurosurgery. 2006;59(1): 157-166.

6. Spinner RJ, Desy NM, Agarwal G, et al. Evidence to support that adventitial cysts, analogous to intraneural ganglion cysts, are also joint-connected. Clin Anat. 2013;26(2):267-281.

7. Spinner RJ, Carmichael SW, Wang $\mathrm{H}$, et al. Patterns of intraneural ganglion cyst descent. Clin Anat. 2008;21(3):233-245.

8. Wilson TJ, Hébert-Blouin MN, Murthy NS, et al. The nearly invisible intraneural cyst: a new and emerging part of the spectrum. Neurosurg Focus. 2017;42(3):E10.

9. Stewart JD. Foot drop: where, why and what to do? Pract Neurol. 2008;8(3):158-169.

10. Stevens F, Weerkamp NJ, Cals JWL. Foot drop. BMJ. 2015;350: h1736.

11. Visser LH. High-resolution sonography of the common peroneal nerve: detection of intraneural ganglia. Neurology. 2006;67(8):1473-1475.

12. Spinner RJ, Atkinson JLD, Tiel RL. Peroneal intraneural ganglia: the importance of the articular branch. A unifying theory. J Neurosurg. 2003;99(2):330-343.

13. Spinner RJ, Desy NM, Amrami KK. The unifying articular (synovial) origin for intraneural ganglion cysts: moving beyond a theory. $J$ Hand Surg Am. 2016;41(7):e223-e224.
14. Amino K, Nagatsuka $Y$, Horibe K, et al. Intraneural ganglion of the peroneal nerve: a case report. Article in Japanese. Kanto $J$ Orthop Traumatol. 1976;7:341-344.

15. Miomo H, Shibata K, Kina M, et al. A case of peroneal nerve paralysis due to intraneural ganglion. Article in Japanese. Orthop Traumatol. 1992;41:180-183.

16. Peng $F$, Yang JY, Yu C, et al. Common peroneal nerve intraneural ganglion cyst: literature review and report of 3 cases. J Anat Clin. 2013;18(4): 298-301.

17. Stack RE, Bianco AJ Jr, MacCarty CS. Compression of the common peroneal nerve by ganglion cysts: report of nine cases. $J$ Bone Joint Surg Am. 1965;47:773-778.

18. Spinner RJ, Hébert-Blouin MN, Amrami KK, Rock MG. Peroneal and tibial intraneural ganglion cysts in the knee region: a technical note. Neurosurgery. 2010;67(3 suppl operative):ons71-ons78.

19. Gardner E. The innervation of the knee joint. Anat Rec. 1948; 101(1):109-130.

20. Murthy NK, Spinner RJ. The paraneurium (circumneurium) and its clinical implications with benign and malignant nerve lesions. Clin Anat. Published online June 16, 2020. doi:10.1002/ca.23639

21. Sim J, Kwak H, Lee S, Min K. Peroneal neuropathy caused by an extraneural ganglion cyst in the supracondylar area of the femur: a case report. Medicine (Baltimore). 2020;99(37):e22123.

22. Spinner RJ, Mokhtarzadeh A, Schiefer TK, et al. The clinico-anatomic explanation for tibial intraneural ganglion cysts arising from the superior tibiofibular joint. Skeletal Radiol. 2007;36(4):281-292.

23. Spinner RJ, Atkinson JLD, Scheithauer BW, et al. Peroneal intraneural ganglia: the importance of the articular branch. Clinical series. J Neurosurg. 2003;99(2):319-329.

24. Wilson TJ, Stone JJ, Howe BM, et al. Joint outcomes following surgery for superior tibiofibular joint-associated peroneal intraneural ganglion cysts. Neurosurgery. 2020;86(3):383-390.

\section{Disclosures}

The authors report no conflict of interest concerning the materials or methods used in this study or the findings specified in this paper.

\section{Author Contributions}

Conception and design: Smith, Amrami. Acquisition of data: all authors. Analysis and interpretation of data: all authors. Drafting the article: Spinner, Smith, Jack, Amrami. Critically revising the article: Spinner, Smith, Jack. Reviewed submitted version of manuscript: Spinner, Smith, Jack, Amrami. Approved the final version of the manuscript on behalf of all authors: Spinner. Study supervision: Spinner.

\section{Correspondence}

Robert J. Spinner: Mayo Clinic, Rochester, MN. spinner.robert@mayo.edu. 